\title{
Treatment with some anti-inflammatory drugs reduces germ tube formation in Candida albicans strains
}

\author{
Elena Rusu ${ }^{1}$, Manuela Radu-Popescu ${ }^{2}$, Diana Pelinescu ${ }^{3}$, Tatiana Vassu ${ }^{3}$ \\ ${ }^{1}$ Preclinical Department, Faculty of Medicine, Titu Maiorescu University, Bucharest, Romania. \\ ${ }^{2}$ Department of Microbiology, Faculty of Pharmacy, University of Medicine and Pharmacy Carol Davila, \\ Bucharest, Romania. \\ ${ }^{3}$ Center for Research, Training \& Consulting in Microbiology, Genetics and Biotechnology, \\ Faculty of Biology, University of Bucharest, Romania
}

Submitted: June 11, 2013; Approved: April 17, 2014

\begin{abstract}
Candida albicans is an opportunistic dimorphic fungus that inhabits various host mucosal sites. It can cause both superficial and serious systemic disease. Conversion from the yeast to the hyphal form has been associated with increased virulence and mucosal invasiveness. The aim of this study was to investigate the effect of sodium diclofenac and aspirin on germs tube formation of different Candida albicans strains. Prostaglandins may play an important role in fungal colonization. Nonsteroidal anti-inflammatory drugs are inhibitors of the cyclooxygenase isoenzymes. These drugs specifically block the biosynthesis of mammalian prostaglandins by inhibiting one or both of cyclooxygenase isoenzymes. In tests for germ tube formation sodium diclofenac reduced the filamentation to the $12.5 \%-5.1 \%$. In the presence of aspirin the filamentation was reduced up to $85-45 \%$ depending on the tested strain. Our results suggest that cyclooxygenase-depending synthesis of fungal prostaglandins is important for morphogenesis and fungal virulence. Inhibitors of cyclooxygenase isoensymes (aspirin and diclofenac) are effective in decreasing germ tube formation of Candida albicans.
\end{abstract}

Key words: Candida, germ tube, diclofenac, aspirin.

\section{Introduction}

Systemic fungal infection have increased dramatically in prevalence and severity over the last few decades, in concert with the number of patients living for extended periods with significant immune dysfunction. AIDS, cancer chemotherapy and organ transplantation have all contributed to this rise, as has the widespread use the antibiotics. The most common systemic fungal infection is candidiasis, which accounts for well over half of these invasive mycoses. A single species, Candida albicans (C. albicans) causes the majority of these infections. Candida albicans, which also causes oropharyngeal thrush and vaginitis, is a normally a commensal of the human gastrointestinal tract, in which it lives without adverse effects on the host. Candida albicans is a polymorphic fungus, and the yeast-to-hypha transition has been associated with increased virulence, mucosal invasiveness and biofilm formation. Candidemia and invasive candidiasis are frequently associated with high morbidity and high mortality rates. C. albicans is a fungus that can exist in three morphotypes: budding yeast, pseudohypha and true hypha. Fungal yeast-mycelium dimorphism is of interest because of the economic and medical importance of dimorphic fungi and because these organisms may serve as model for studying differentiation. These yeasts often cause opportunistic fungal infection in human patients who have become immune-compromised by anticancer therapy, HIV infection, organ transplantation or therapy with broad-spectrum antibiotics, leading to sever fungal infection (Washington et al., 2006). The physiological and immune condition of the host and the yeasts adaptation in surviving in many anatom- 
ical sites are important factors in the transition from commensally to disease-causing yeasts. Many putative virulence factors can contribute to the yeasts invasiveness and pathogenicity, such as their ability, conversion of unicellular yeasts into filamentous forms and expression of extracellular enzymes. Several antifungal drugs, such as fluconazole, ketoconazole, nystatin, amphotericin $\mathrm{B}$ and 5-fluorocytosine can interfere with virulence factors.

In recent years, due to the increased resistance of many bacteria to the commonly used antimicrobial agents, attention has shifted to drugs belonging to different pharmacological classes for possible antimicrobial activity. This, borne out of the fact that a single drug may have varying properties with diverse physiological activities and functions and as a results, may have useful activities in completely different spheres of medicine. This, in turn, has led to the investigation of drugs belonging to different pharmacological classes for possible antimicrobial activity. The prophylactic and curative treatments with antifungal drugs can cause the appearance of Candida resistant-strains. Infection by $C$. albicans of host tissue and cell is mediated through surface receptors, such as mannose, $\beta$-glucans, integrins and had been found to release pro-inflammatory cytokines and large amount of arachidonic acid (AA) from host cell. AA is subsenquently converted by lipooxygenases and cyclooxygenases (COXs) to eicosanoids (Noverr et al., 2001). Prostaglandins (PG) are lipid molecules and may play an important role in fungal colonization. Nonsteroidal anti-inflammatory drugs (sodium diclofenac, aspirin) are inhibitors of the cyclooxygenase isoenzymes (COX-1 and COX-2). These drugs specifically block the biosynthesis of mammalian prostaglandins by inhibiting one or both of COX isoenzymes. Because $C$. albicans has been reported to produce prostaglandins $\mathrm{E}_{2}\left(\mathrm{PGE}_{2}\right)$ in $\mathrm{HeLa}$ cells (Deva et al., 2001) and $\mathrm{PGE}_{2}$ has been shown to induce germ tubes formation in C. albicans ((Kalo-Klein et al., 1990), a vicious cycle seems to be operative in mucocutaneous candidiasis, in which the infected host tissue releases $\mathrm{AA}$ and $\mathrm{PGE}_{2}$.

The aim of this study was to investigate the effect of sodium diclofenac and aspirin on germs tube formation of different Candida albicans strains. The model HeLa cells/C. albicans closely represented the vulvovaginal candidiasis (Deva et al., 2001).

\section{Material and Methods}

\section{Yeast strains}

Five strains of Candida albicans were used in this study. Candida strains C1 and C4 were isolated at Sfantul Pantelimon Hospital, Bucharest, from the sputum of two patients and C2, C3, and C5 strains were isolated at Matei Bals Hospital, Bucharest, from the oropharyngean excreta of three patients. Clinical isolates were first characterized and taxonomically identified based on colony morphology and microscopical appearance. The identification studies had been completed by the yeast API 20 C AUX test (bioMerieux, France). Yeasts were initially isolated on YPG agar, stored in glycerol, and frozen at $-70{ }^{\circ} \mathrm{C}$ until analysis. We used also reference strain Candida albicans ATCC (American Type Culture Collection) 10231.

\section{Medium and culture conditions}

Strains were grown in Yeast Peptone Glucose (1\% yeast, $2 \%$ peptone and $2 \%$ glucose) medium (YPG). Batches of medium $(10 \mathrm{~mL})$ were inoculated from the fresh culture slopes and incubated at $37{ }^{\circ} \mathrm{C}$ for $24 \mathrm{~h}$. All strains grew exclusively in the budding yeast phase under these conditions. Before used in experiments, cell suspensions were standardized to a cell density of $0.5 \mathrm{McF}$ arland.

\section{COX inhibitors}

Stock solutions $(100 \mathrm{mM})$ of sodium diclofenac and aspirin (acetylsalicylic acid) were prepared in ethanol.

\section{Germ tube tests}

Cultures of Candida albicans strains were grown overnight in YPG-medium. For this experiment we used a HeLa cell line for determination the ability of Candida cell to form germ tubes. The cells were resuspended in the potassium phosphate buffer $(\mathrm{pH}=7.4)$ and placed on the HeLa cell line. COX inhibitors were added to a final concentration of $0.1 \mathrm{mM}$. HeLa cells with Candida albicans suspensions were incubated at $37{ }^{\circ} \mathrm{C}$ for $1.5 \mathrm{~h}$. For visualization we used Giemsa solution. For examination the percentage of germ tubes present we used a light microscope; 100 cells were counted each time.

\section{Results and Discussions}

In recent years, due to the increased resistance of many bacteria to the commonly used antimicrobial agents, attention has shifted to drugs belonging to different pharmacological classes for possible antimicrobial activity. A number of registered non-antibiotic drugs possess antimicrobial effect that have generally been regarded as a side-effect, as in the case with anti-diuretic, anti-diabetic, $\beta$-blocker, and non-steroidal anti-inflammatory molecules (Cedurlund et al., 1993). Morphogenesis, the transition of unicellular yeasts cells to the filamentous form, is an attribute of Candida albicans species. C. albicans is a fungus that can exist in three morphotypes: budding yeast, pseudohypha and true hypha. The presence of the filamentous form and budding is associated with virulence and pathogenicity, but both forms may be involved in the development and progress of disease. C. albicans is able to develop single spherical cells including typical yeast cells and chlamydospores, as well as elongated cells developing into multicellular true hypha or pseudohypha. Thus, the term dimorphism, which traditionally is reserved for the yeast-true 
hypha inter-conversion, in a more general sense designates the main theme of C. albicans and possibly fungal morphogenesis in general. Budding-yeast cells can be induced to form true hypha, which grow by continuous apical extension followed by septation. Pseudohypha grew differently from true hypha, by unipolar budding: buds develop into elongated cells, which remain attached to mother cells, stop growth and resume budding. Some environmental factors have been reported as determinants of morphological regulation, particularly in C. albicans. For instance, hyphainducing conditions include stimuli such as an increase in temperature, in $\mathrm{pH}$ level, in serum, nutrient starvation, and in cell density (Ernst, 2000). Exogenous $\mathrm{PGE}_{2}$ from either host or fungal sources enhances germ tube formation in $C$. albicans, implicating fungal eicosanoids as a morphogenic factor (Noverr et al., 2001). Prostaglandins production could be inhibited by aspirin, which also suppresses the growth of the yeast form and prevents the yeast to hypha transition of Candida albicans. The yeast to hypha transition is often associated with progression of infection by $C$. albicans, and therefore, fungal eicosanoids regulation of morphogenesis may be considered a virulence mechanism. Sodium diclofenac and aspirin specifically block the biosynthesis of mammalian prostaglandins by inhibiting one or both of COX isoenzymes.

During Candida albicans infection, AA is released from phospholipids of infected host cells by fungal phospholipases (Deva et al., 2000). C. albicans utilizes the released AA as the sole carbon source for cell growth and morphogenesis. $C$. albicans has been reported to produce $\mathrm{PGE}_{2}$ in HeLa cells (Deva et al., 2001) and $\mathrm{PGE}_{2}$ has been shown to induce germ tubes formation in $C$. albicans (Kalo-Klein et al., 1990). For the production of germ tubes, the diclofenac registred an important inhibition effect on $C$. albicans cells (Figure 1).

In the presence of diclofenac sodium $(0.1 \mathrm{mM})$, the filamentation was reduced to the $12.5 \%-5.1 \%$. Germ tubes formation is expressed as a percentage of that for control cells incubated in the absence of COX inhibitors (Figure 2). Aspirin reduced the germ tube formation of tested C. albicans strains up to $86 \%-45 \%$. Previous authors studied the role of diclofenac in the dimorphic transition in $C$. albicans. Their results indicated the effect of diclofenac was dependent on the concentration of this compound and preincubation with $500 \mu \mathrm{g} / \mathrm{mL}$ diclofenac completely inhibited hypha formation in liquid and solid media (Ghalehnoo et al., 2010). Effect of aspirin and piroxicam of cell viability on Candida species was also studied (Rusu et al., 2009). Piroxicam drastically reduced the viability of planktonic cells to $0.94 \%$ and aspirin had reduced to the $4.45 \%$ of that of untreated control cells.

Aspirin and diclofenac inhibit both the COX-1 and COX-2 isoenzymes. The COX active site is created by a long hydrophobic channel that is also the site of drug binding. Aspirin is the only known non-steroidal anti-inflam-
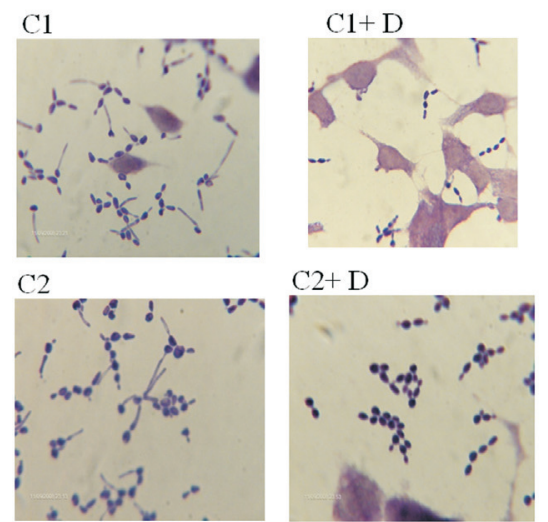

C3
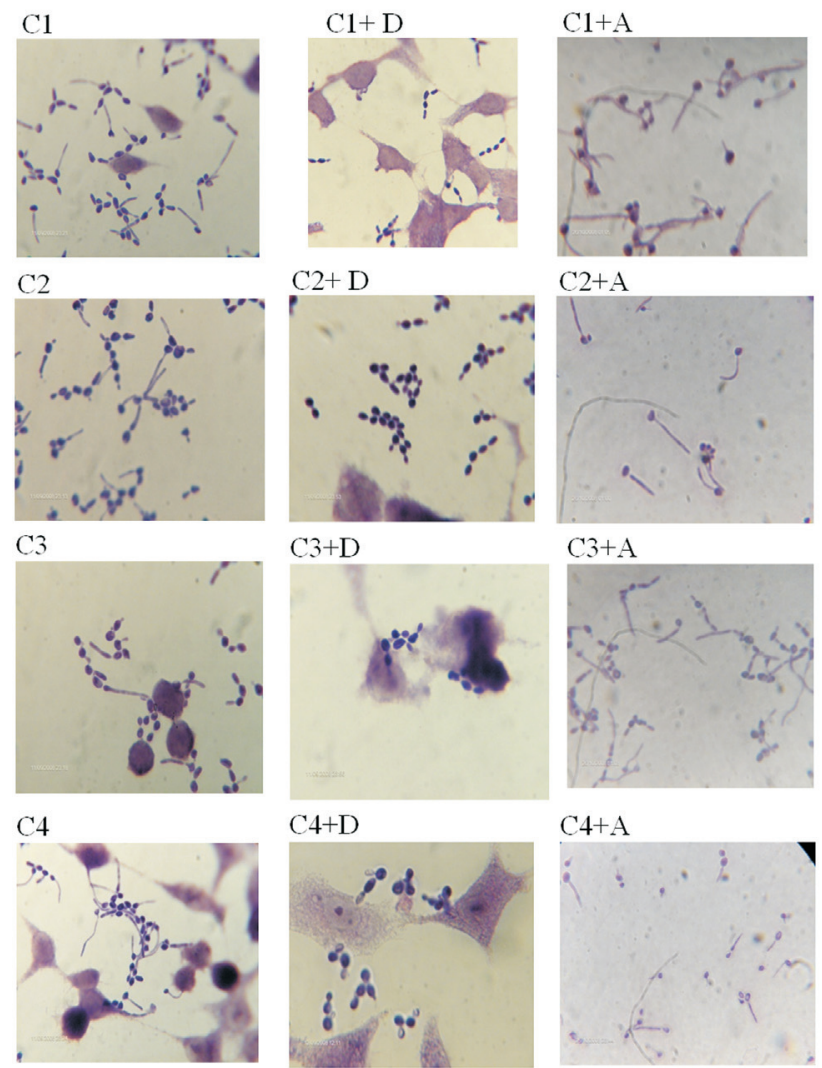

C5
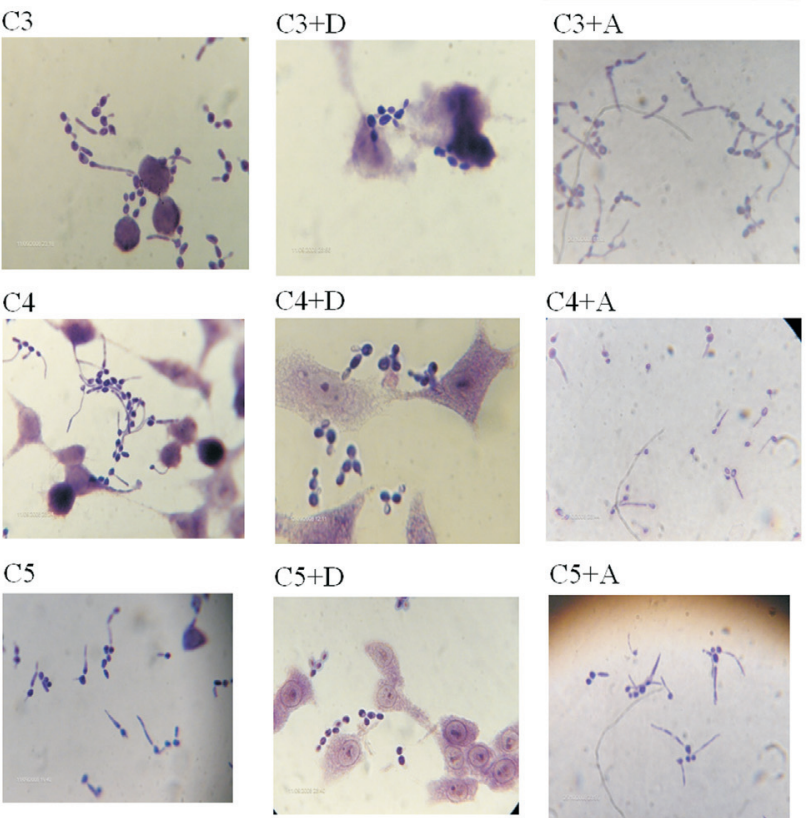

C. albicans ATCC

10231

C.albicans ATCC $10231+\mathrm{D}$
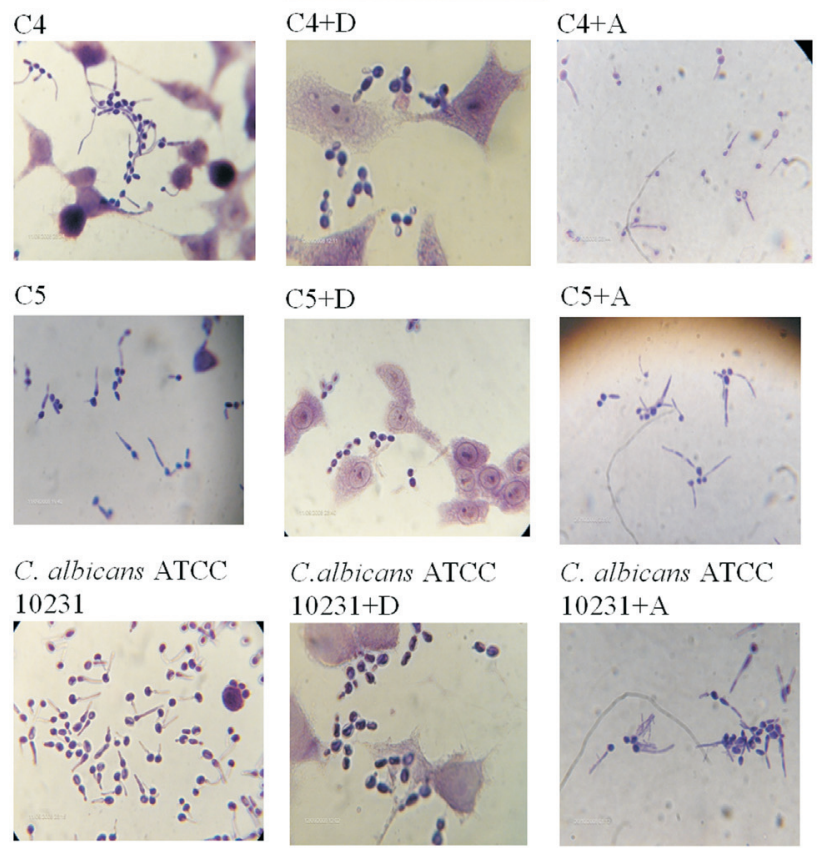

$\mathrm{C} 5+\mathrm{A}$

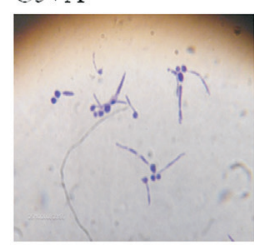

C. albicans ATCC $10231+\mathrm{A}$

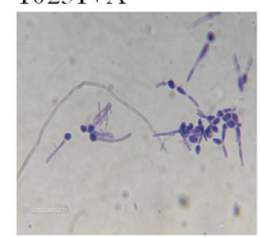

Figure 1 - Influence of sodium diclofenac (D) and aspirin(A) on Candida albicans strains morphology $(0.1 \mathrm{mM})$ on HeLa line after $2 \mathrm{~h}$ at $37^{\circ} \mathrm{C}$.

matory drug that covalently binds to a serine residue and that inhibits COX-1 more than COX-2. It is thought that COX-1 is expressed constitutively in most tissues of the body and COX-2 is mainly an inducible enzyme involved in regulation of inflammation (Dutta et al., 2007). COX-1 plays important roles beyond thromboxane $\mathrm{A}_{2}\left(\mathrm{TXA}_{2}\right)$ production in platelets. COX-2, a second cyclooxygenase isoenzyme primarily responsible for synthesis of the platelet inhibitors $\mathrm{PGI}_{2}$ by endothelial cells is induced in response to inflammatory stimuli, and is less sensitive to the effects of aspirin (Cheng et al., 2006). The inducing stimuli include pro-inflammatory cytokines and growth factors, 


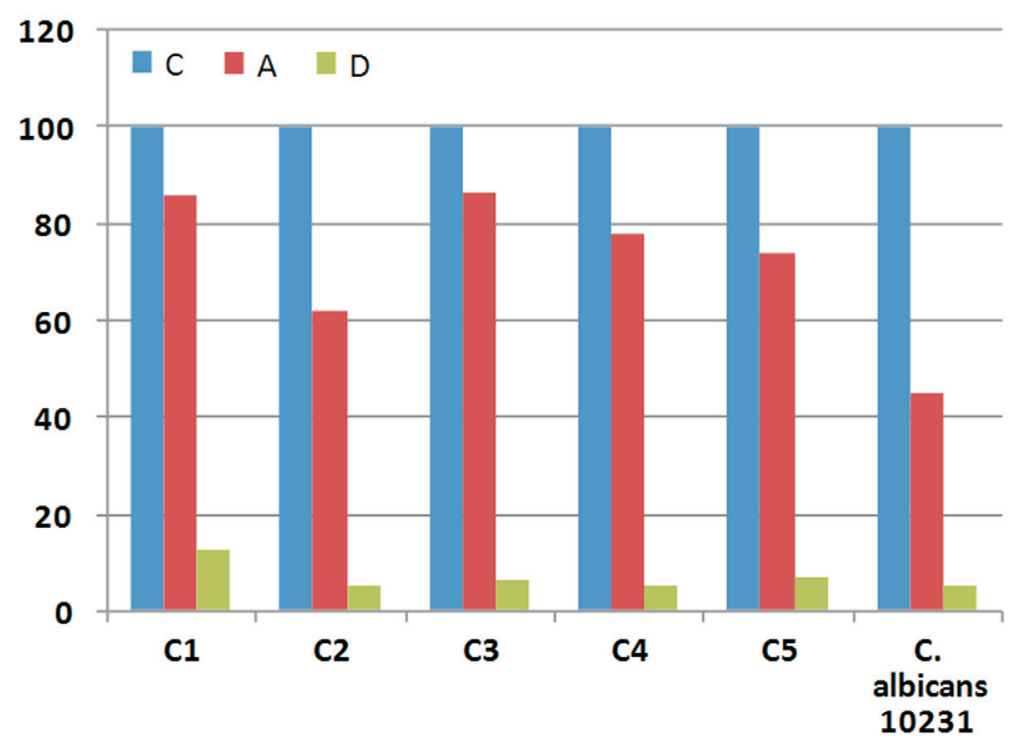

Figure 2 - Effects of sodium diclofenac (D) and aspirin (A) on germ tube formation by Candida albicans strains. Germ tube formation is expressed as a percentage of that for control cells $(\mathrm{C})$ incubated in the absence of COX inhibitors (final concentration $0.1 \mathrm{mM}$ ).

implying COX-2 in both inflammation and control of cells growth (Vane et al., 1998). In this study, two COX inhibitors tested decreased germ tubes of $C$. albicans with aspirin and diclofenac, a preferential COX-2 inhibitor, producing the greatest effects. Diclofenac has a low to moderate preference to block the COX-2 isoensyme (approximately 10 fold) and have a somewhat lower incidence of gastrointestinal complaints than noted with aspirin. Diclofenac has recently been discovered to inhibit microbial biofilms. A biofilm is a population of cells growing on a surface and enclosed in an exopolysaccharides matrix. Biofilms confer resistance on micro-organisms to antibiotic treatment. The development of resistance by microorganisms to antimicrobial drugs has been one of the greatest problems hampering antimicrobial therapy. Bacterial biofilms show enormous levels of antibiotic resistance, which is a general feature of all biofilms. Sodium diclofenac has remarkable inhibitory action both against drug-sensitive and drugresistant clinical isolates of various Gram-positive and Gram-negative bacteria. Dutta N. K. and his colleagues had determinate the ability of diclofenac to protect mice from a virulent Salmonella infection. Their study had demonstrated that diclofenac $(1.5-3 \mu \mathrm{g} / \mathrm{g})$ protected animals from the lethality of Salmonella (Dutta et al., 2007). In another study, aspirin or ibuprofen was administered to mice undergoing treatment of tuberculosis infection (Mycobacterium tuberculosis) to determine if these non-steroidal anti-inflammatory drugs enhance pyrazinamide activity in vivo (Byrne et al., 2007). The results shown aspirin and ibuprofen enhance the effect of pyrazinamide during the initial phase of tuberculosis treatment in the mouse model.

There is some evidence that diclofenac inhibits the lipooxygenase pathways, thus reducing formation of the leukotrienes. Also, diclofenac may inhibit phospholipase
$\mathrm{A}_{2}$ as part of its mechanism of action. Phospholipase $\mathrm{A}_{-2}$ and phospholipase $\mathrm{B}$ have been identified in a large number of eukaryotic microbes, including Candida albicans, Cryptococcus neoformans and Aspergillus fumigatus. Phospholipases A and B cleave the fatty acid side chains of phospholipids have been implicated in virulence in a number of parasitic and antifungal species, presumably via destruction of host cell membranes and subsequent lysis (Noverr et al., 2003). These additional actions may explain the high potency of diclofenac. Another non-steroidal antiinflammatory drug, ibuprofen, was described as being able to reverts resistance related to efflux activity in C. albicans (Pina-Vaz et al., 2000). Fluconazole resistant isolates revering to susceptibility after incubation with ibuprofen showed $C D R 1$ and $C D R 2$ genes overexpresion especially of the latter (Ricardo et al., 2009).

The increasing frequency of invasive fungal infections and the high mortality rate associated with disseminated fungal diseases have underscored the importance of finding new therapy or improving for fungal infections. Inhibitors of cyclooxygenase isoensymes (aspirin and diclofenac) are effective in decreasing germ tube formation of Candida albicans. Sodium diclofenac had the greatest effects on Candida albicans strains. Nonsteroidal antiinflammatory drugs specifically block the biosynthesis of fungal prostaglandins may be one strategy to combat fungal colonization and infection. Our results suggest that cyclooxygenase-depending synthesis of fungal prostaglandins is important for morphogenesis and the survival of Candida albicans cells and may act as a regulator in these processes. The analgesic and anti-inflammatory properties of aspirin and diclofenac might represent an additional advantage for their use in the management of infections with Candida albicans. 


\section{References}

Byrne ST, Denkin SM, Zhang Y (2007) Aspirin and ibuprofen enhance pyrazinamide treatment of murine tuberculosis. J Antimicrob Chemother 59: 313-316.

Cedurlund H, Mardh PA (1993) Antibacterial activities of nonantibiotic drugs. J Antimicrob Chemother 32:355-365.

Cheng Y, Wang M, Yu Y, Lawson J, Funk CD, Fitzgerald GA (2006) Cyclooxygenase, microsomal prostaglandin E synthase-1, and cardiovascular function. J Clin Invest 116:1391-1399.

Deva R, Ciccoli R, Schewe T (2000) Arachidonic acid stimulates cell growth and forms a novel oxygenated metabolite in Candida albicans. Biochim Acta 1486:299-311.

Deva R, Ciccoli R, Kock L, Nigam S (2001) Involvement of aspirin-sensitive oxylipins in vulvovaginal candidiasis. FEMS Microbiol Lett 198:37-43.

Dutta KN, Annadurai S, Mazumdar K, Dastidar S, Kristiansen J, Molnar J, Martins M, Amaral L (2007) Potential management of resistant microbial infections with a novel nonantibiotic: The anti-inflammatory drug diclofenac sodium. Int J Antimicrobial Agents 30:242-249.

Ernst JF (2000) Transcription factors in Candida albicans: Environmental control of morphogenesis. Microbiology 146:1763-1774.

Ghalehnoo ZR, Rashki A, Najimi M, Dominguez A (2010) The role of sodium diclofenac in the dimorphic transition in Candida albicans. Microb Pathog 48:110-115.
Kalo-Klein A, Witkin SS (1990) Prostaglandin $E_{2}$ enhances and $\gamma$ interferon inhibits germ tube formation in Candida albicans. Infect Immunity 58:260-262.

Noverr MC, Phare SM, Towes BG, Coffey MJ, Huffnagle GB (2001) Pathogenic yeasts Cryptococcus neoformans and Candida albicans produce immunomodulatory prostaglandins. Infect Immunity 69:2957-2963.

Noverr MC, Erb-Downward JR, Huffnagle GB (2003) Production of eicosanoids and other oxylipins by pathogenic eukaryotic microbes. Clin Microbiol Rev 16:517-533.

Pina-Vaz C, Sansonetti F, Rodrigues AG, Martinez De Oliveira J, Fonseca AF Mardh PA (2000) Antifungal activity of ibuprofen alone and in combination with fluconazole against Candida species. J Med Microbiol 49:831-840.

Ricardo E, Costa-de-Oliveira S, Dias AS, Guerra J, Rodriquez AG, Pina-Vaz C (2009). Ibuprofen reverts antifungal resistance on Candida albicans showing overexpression of CDR genes. FEMS Yeast Res 9:618-625.

Rusu E, Vassu T, Enache-Soare S (2009) Aspirin and piroxicam effects on cellular viability of some Candida strains. Ro J Stomatol LV:60-64.

Vane JR, Bachle Y S, Botting RM (1998) Cyclooxygenase 1 and 2. Annu Rev Pharmacol Toxicol 38:97-120.

Washington W, Stephen A, William J, Koneman EW, Schreckenberger PC (2006) Koneman's Color Atlas and Textbook of Diagnostic Microbiology. 6th edition. Lippincott Williams \& Williams, Philadelphia, 1736 pp.

All the content of the journal, except where otherwise noted, is licensed under a Creative Commons License CC BY-NC. 